\title{
ÉTUDE DE LA STRUCTURE OVARIENNE DE BARBUS HOLOTAENIA, BOULENGER, 1904 (PISCES, CYPRINIDAE) DU BASSIN DE LA LUKI (ZAIIRE).
}

\section{S. MUTAMBUE}

\begin{abstract}
Département de Biologie Chimie, Institut Supérieur Pédagogique Mbanza-Ngungu,
\end{abstract} B.P. 127, Mbanza-Ngungu, République du Zaïre.

\section{RÉSUMÉ}

L'examen histologique et cytologique des ovaires de Barbus holotaenia a permis de distinguer différents stades de développement (stades 0 à VI). Le stade 0 correspond à la transformation des cellules primordiales en ovocytes. Le stade I est représenté par de petites cellules avec un gros noyau central (ovocytes I). Le stáde II correspond au stade périnucléolaire (plusieurs nucléoles accolés à la membrane nucléaire). Les stades III à $\mathrm{V}$ sont caractérisés par l'accumulation de vitellus (prévitellogenèse endogène et vitellogenèse exogène). Le stade VI marque le début de la phase de repos avec l'atrésie des follicules. L'observation d'ovocytes I en vitellogenèse et d'alevins pendant la saison des pluies permet de penser que la vitellogenèse et la ponte se déroulent à cette époque de l'année.

Mots-clés : Zaïre, Barbus holotaenia, structure ovarienne, vitellogenèse, ponte, étude histologique.

\section{STUDY OF OVARIAN STRUCTURE IN BARBUS HOLOTAENIA, BOULENGER, 1904 (PISCES, CYPRINIDAE) FROM THE LUKI RIVER BASIN (ZAÏRE).}

\section{ABSTRACT}

The histological and cytological analysis of the ovaries of Barbus holotaenia has allowed to distinguish different developmental stages (stages 0 to $\mathrm{V} !$ ) : stage 0 corresponding to the development of germinal cells in oocytes I, stade I showing small cells with a large central nucleus, stage II distinguished by a number of nucleolus on the periphery of the nuclear membrane, stages III to $V$ characterized by the deposition of yolk and stage VI showing atretic follicles. The observation of advanced stages of ovogenesis and fry during rainy season shows that vitellogenesis and spawning occur in this period.

Key-words : Zaïre, Barbus holotaenia, ovarian structure, vitellogenesis, spawning, histological analysis.

\section{INTRODUCTION}

En zone tempérée, la description du cycle de reproduction des poissons dulcicoles et/ou marins a fait l'objet de nombreux travaux. En Afrique, ces études sont rares et ponctuelles surtout dans le domaine dulcicole. Au Zaïre, ces études sont intégrées dans des. recherches biologiques, éthologiques et écologiques menées dans le cadre de projets d'élevage chez les Cichlidae (Oreochromis niloticus, LINNAEUS, 1758 ; Tilapia macrochir, BOULENGER, 1912 ; T. melanopleura, DUMERIL, 1859 et $T$. mossambicus, PETERS, 1852) dont la valeur commerciale et la capacité de reproduction sont importantes (DE BONT, 1948, 1950 ; RUWET, 1962, 1963, 1968 ; DESTESCHE-GOMEZ et RUWET, 1967 ; FRANSSEN et LAPERCHE, 1980). D'autres aspects de la reproduction tels que la taille à la première maturation, le lieu et la période de ponte de bon nombre d'espèces recensées dans le milieu naturel ont été également abordés (GOSSE, 1963 ; MATTHES, 1964 ; DE KIMPE, 1964 ; MUTAMBUE, 1984, 1992). 
Les mécanismes impliqués dans le contrôle du cycle de reproduction des poissons téléostéens sont variés et se prêtent peu à la généralisation en raison de la diversité des biotopes dans lesquels ils vivent (BILLARD et BRETON, 1978 ; LEGENDRE et JALABERT, 1988). Chez les espèces tropicales, dont la reproduction est limitée dans le temps, l'influence de la crue apparaît prépondérante puisqu'elle coïncide avec la dernière phase de la gamétogenèse et la ponte. Dans le cas d'espèces dont la reproduction est continue, la période de crue peut même correspondre à un pic de reproduction (DAGET, 1954 ; GOSSE, 1963 ; MATTHES, 1964 ; DE KIMPE, 1964 ; LOWE MC CONNEL, 1964 ; ALBARET, 1982 ; BENECH et QUENSIERE, 1985). La température et la photopériode ne sont pas significativement liées à cette saison (BENECH et QUENSIERE, 1985). II est possible que ces deux facteurs écologiques ne jouent pas un rôle essentiel dans l'induction de la maturation ovarienne et de la ponte des espèces tropicales comme c'est le cas chez les espèces des régions tempérées (KIRSCHBAUM, 1984 ; BENECH et QUENSIERE, 1985). Jusqu'à présent, l'influence d'autres paramètres tels que le $\mathrm{pH}$, la concentration en ions et la nébulosité a été moins étudiée en raison de leur forte variabilité d'une région à l'autre. En fait, faute d'expérimentation, il est difficile à l'heure actuelle de définir l'implication exacte de tous ces facteurs dans la stimulation, l'inhibition ou la régularisation des différentes phases du cycle de reproduction des poissons tropicaux.

Dans ce travail, nous décrivons les changements qui se produisent dans les ovaires de Barbus holotaenia du bassin de la rivière Luki au cours de leur cycle de reproduction.

\section{MATÉRIEL ET MÉTHODES}

Cette étude a porté sur 156 femelles pêchées mensuellement de juillet 1986 à août 1987, puis trimestriellement de septembre 1987 à septembre 1988. Les sites de capture sur la rivière Luki $\left(L_{1}\right.$ à $\left.L_{5}\right)$ et sur ses affluents $\left(A_{1}\right.$ à $\left.A_{5}\right)$ sont indiquées à la figure 1 .

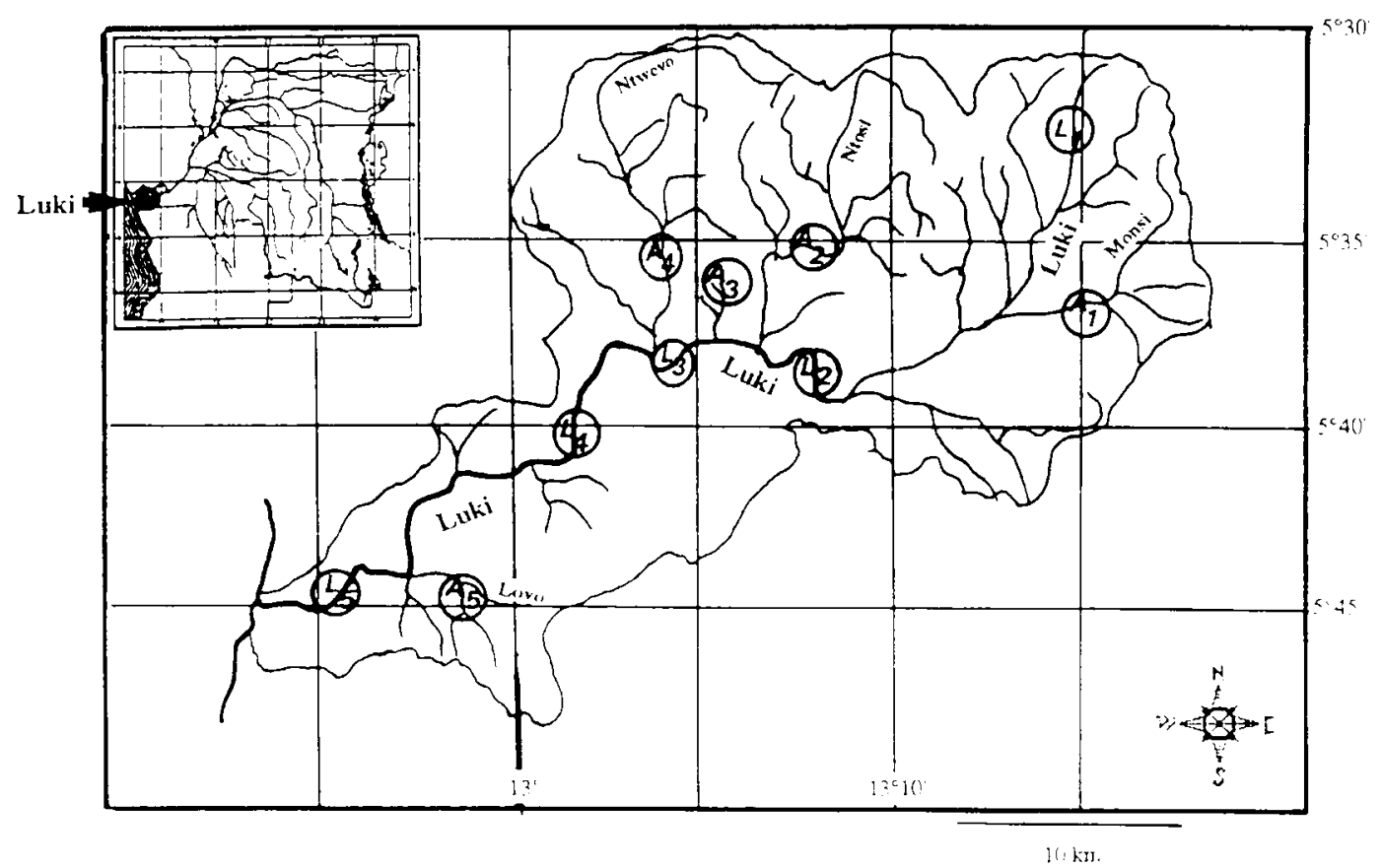

Figure 1 : Situation géographique du bassin de la Luki. Localisation des sites de capture sur la Luki $\left(L_{1}\right.$ à $\left.L_{5}\right)$ et ses affluents $\left(A_{1}\right.$ à $\left.A_{5}\right)$.

Figure 1 : Geographic situation of the Luki river basin. Localisation of catching sites in Luki river $\left(L_{1}\right.$ to $\left.L_{5}\right)$ and its tributaries $\left(A_{1}\right.$ to $\left.A_{5}\right)$. 
Nous avons mesuré le poids corporel $(\mathrm{g})$ et la longueur standard $(\mathrm{mm})$ de chaque poisson. Les ovaires ont été pesés, fixés dans une solution de Bouin, déshydratés et inclus dans la paraffine.

Les différents stades de développement des ovaires ont été observés à partir de coupes histologiques de 5 à $7 \mu \mathrm{m}$ d'épaisseur colorées à l'Azan de ROMEIS et au trichrome de MASSON-GOLDNER. La coloration au bleu alcian $(\mathrm{pH} \mathrm{2,5)}$ ainsi que les réactions signalétiques d'HARTIG-ZHAKARIAS et P.A.S. ont été utilisées pour préciser la nature chimique de certains constituants du cytoplasme. La combinaison de la coloration au bleu Alcian ( $\mathrm{pH} \mathrm{2,5)} \mathrm{et} \mathrm{de} \mathrm{la} \mathrm{réaction} \mathrm{P.A.S.} \mathrm{a} \mathrm{permis} \mathrm{de-distinguer} \mathrm{les} \mathrm{polysaccharides}$ porteurs de groupements acides de polysaccharides neutres; la coloration de MANN$D O M I N I C l$ a été utilisée pour mettre en évidence les zones acidophiles et basophiles.

Les changements cycliques des gonades ont été déterminés à l'aide de l'indice gonadosomatique moyen mensuel (I.G.S.), calculé de juillet 1986 à août 1987, selon la formule suivante (BOUGIS, 1952 ; ABOUSSOUAN et LAHAYE, 1979 ; KHALAF, 1987):

$$
\text { I.G.S. }=100 \times(\mathrm{Pg} / \mathrm{P})
$$

où $\mathrm{Pg}=$ poids des gonades et $\mathrm{P}=$ poids corporel total.

La terminologie adoptée dans la description de ces ovocytes s'inspire de celles utilisées par BOUGIS (1952), STAHL et LERAY (1961), et DURAND et LOUBENS (1970).

\section{RÉSULTATS}

\section{Morphologie des ovaires}

Les gonades des femelles observées (poids corporel moyen : $12,3 \mathrm{~g} \pm 5,7 \mathrm{~g}$; longueur corporelle moyenne : $65,3 \mathrm{~mm} \pm 7,8 \mathrm{~mm}$ ) ont une taille variant de 5,5 à $15 \mathrm{~mm}$ $(\bar{X}=9,9 \mathrm{~mm} \pm 2,88 \mathrm{~mm})$ et un poids allant de 0,1 à $3,96 \mathrm{~g}(\bar{X}=1,402 \pm 0,95)$. En période de reproduction, les ovaires deviennent compacts et leur surface granuleuse. En période de repos, ils forment des masses flasques dont la tunique externe est plissée.

La figure 2 représente les variations mensuelles moyennes de l'indice gonadosomatique (I.G.S.). Le cycle sexuel de Barbus holotaenia passe par trois phases principales. Une première phase de maturation gonadique s'étend de la fin du mois d'août au mois de novembre, au cours de laquelle l'I.G.S. croît jusqu'à une valeur maximale moyenne de $26 \%$. Une deuxième phase dite de ponte commence en décembre et se termine vers la première quinzaine de mai, au cours de laquelle l'I.G.S. décroît pour atteindre en fin de période la valeur moyenne de $7,2 \%$. Une troisième période dite "phase de repos" (atrésie des follicules) s'étend de la deuxième quinzaine de mai à la mi-septembre, période pendant laquelle l'I.G.S. moyen ( $3,7 \%$ ) est minimal avec de faibles fluctuations.

\section{Détermination des stades de développement des follicules}

Les ovaires de $B$. holotaenia présentent des follicules à différents stades de développement en période de maturation. Pour classer ces follicules, nous nous sommes basés sur la taille et sur l'aspect du cytoplasme et du noyau des ovocytes ainsi que sur la structure des enveloppes les entourant. Six stades de développement ont pu être ainsi définis :

Stade 0 - Ce stade comportant les différentes étapes de transformation des cellules germinales en ovocytes n'a pas été observé.

Stade I ou stade protoplasmique - A ce stade, les ovocytes sont petits (40 à $90 \mu \mathrm{m}$ ). Le cytoplasme est homogène, très basophile et coloré en noir violacé par l'hématoxyline de Groat. La membrane de l'ovocyte est simple. Le noyau, de forme variable, contient un nucléole primaire basophile et plusieurs autres plus petits. Le rapport diamètre du noyau / diamètre de l'ovocyte $(d / D)$ est de 0,62 $\pm 0,13$.

Stade II ou stade périnucléolaire (Fig. 3A) - Les ovocytes mesurent de 91 à $350 \mu \mathrm{m}$. Le cytoplasme contient de nombreuses granulations intensément colorées par l'hématoxyline de Groat. Les nucléoles sont accolés à la membrane du noyau de forme sphérique. A ce stade, apparaît dans le cytoplasme une zone sphérique très basophile appelée «noyau de Balbiani». Le rapport d/D est de $0,40 \pm 0,01$. 


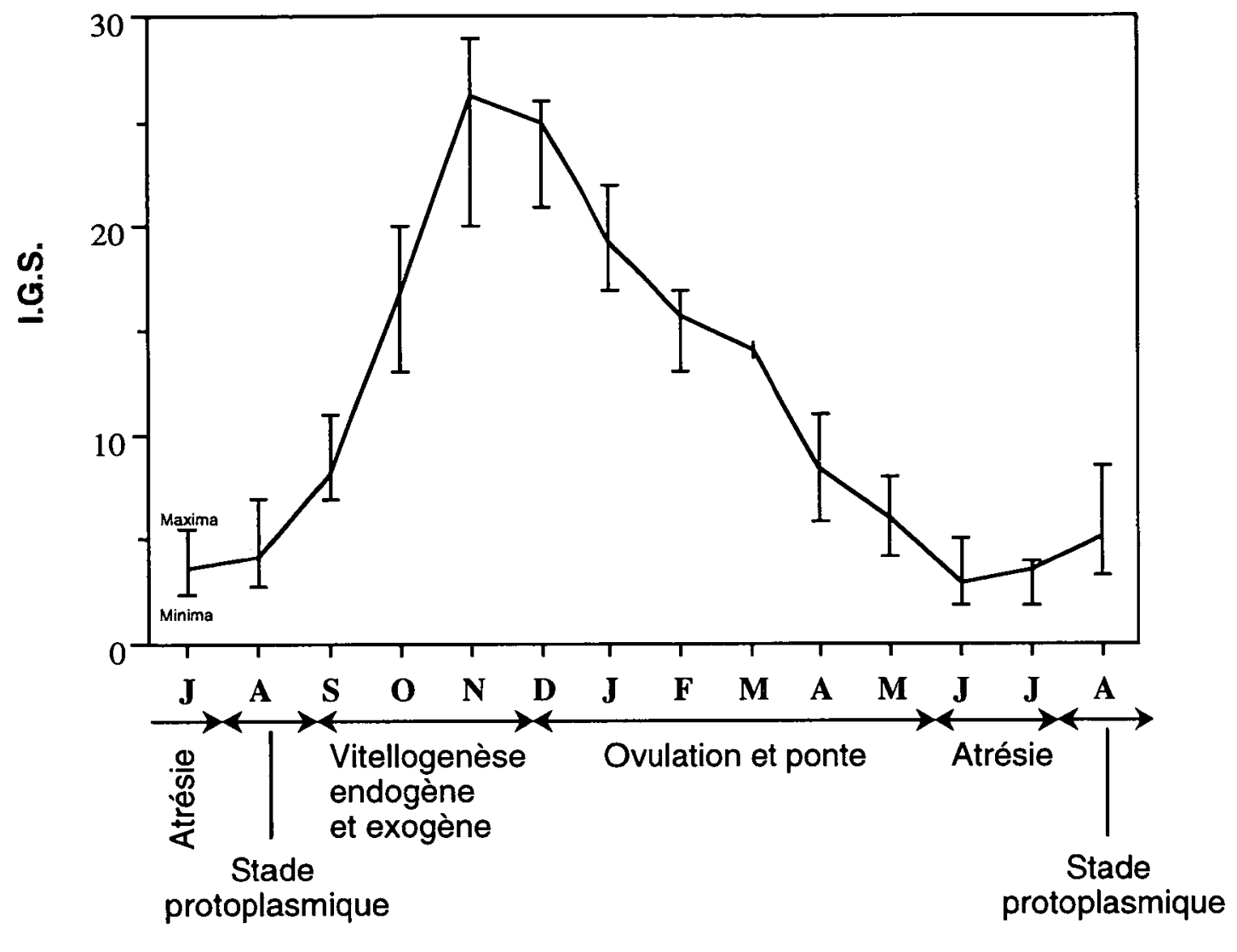

1986

Figure 2 : Evolution de l'indice gonado-somatique en fonction des stades de développement des follicules ovariens chez Barbus holotaenia.

Figure 2 : Evolution of gonadosomatic index mean according to stages of follicle ovarian development in Barbus holotaenia.

Stade III ou stade prévitellogénique (Fig. 3B) - Le diamètre des ovocytes se situe entre 351 et $700 \mu \mathrm{m}$. La périphérie de l'ovocyte est occupée par une rangée des vacuoles corticales (vésicules vitellines). La coloration au bleu de alcian-P.A.S. révèle la présence de polysaccharides acides dans certaines de ces vacuoles colorées en bleu alors que d'autres, roses, contiennent des glycoprotéines. La membrane de l'ovocyte se différencie en membranes folliculaires (thèque et granulosa) et en membranes ovocytaires (membrane vitelline et zona radiata). La membrane vitelline est P.A.S. positive. La zona radiata se présente sous l'aspect d'une zone hyaline acidophile. Le rapport d/D moyen est de 2,6 $\pm 0,06$. La formation d'inclusions lipidiques dans la zone périnucléaire et de microvillosités folliculaires marque le début de la vitellogenèse exogène (accumulation de vitellus proprement dit). Ces inclusions lipidiques fusionnent pour former des globules ou plaquettes vitellines de nature lipoprotéique.

Stade IV ou stade vitellogénique (Fig. 3C) - A ce stade, les ovocytes ont une taille qui varie entre 701 et $960 \mu \mathrm{m}$. Les inclusions lipidiques et les plaques vitellines envahissent le cytoplasme du centre vers la périphérie en repoussant les vacuoles corticales jusqu'à ce qu'elles ne forment plus qu'une seule rangée. Le noyau devient irrégulier et les nucléoles se dispersent dans le nucléoplasme. 
Stade V ou ovocytes mûrs (Fig. 3D) - Les ovocytes de forme polyédrique ont atteint la taille maximale $(961$ à $1100 \mu \mathrm{m})$. Le cytoplasme est entièrement rempli par les plaquettes vitellines et les inclusions lipidiques. Le noyau n'est plus visible.

Stade VI ou atrésie - Après ovulation et ponte, les follicules vides forment des masses flasques souvent jaunâtres. Ceux qui renferment encore des ovocytes en fin de maturation seront progressivement résorbés. Ainsi, à la fin du cycle de reproduction, l'ovaire entre dans sa phase de récupération.
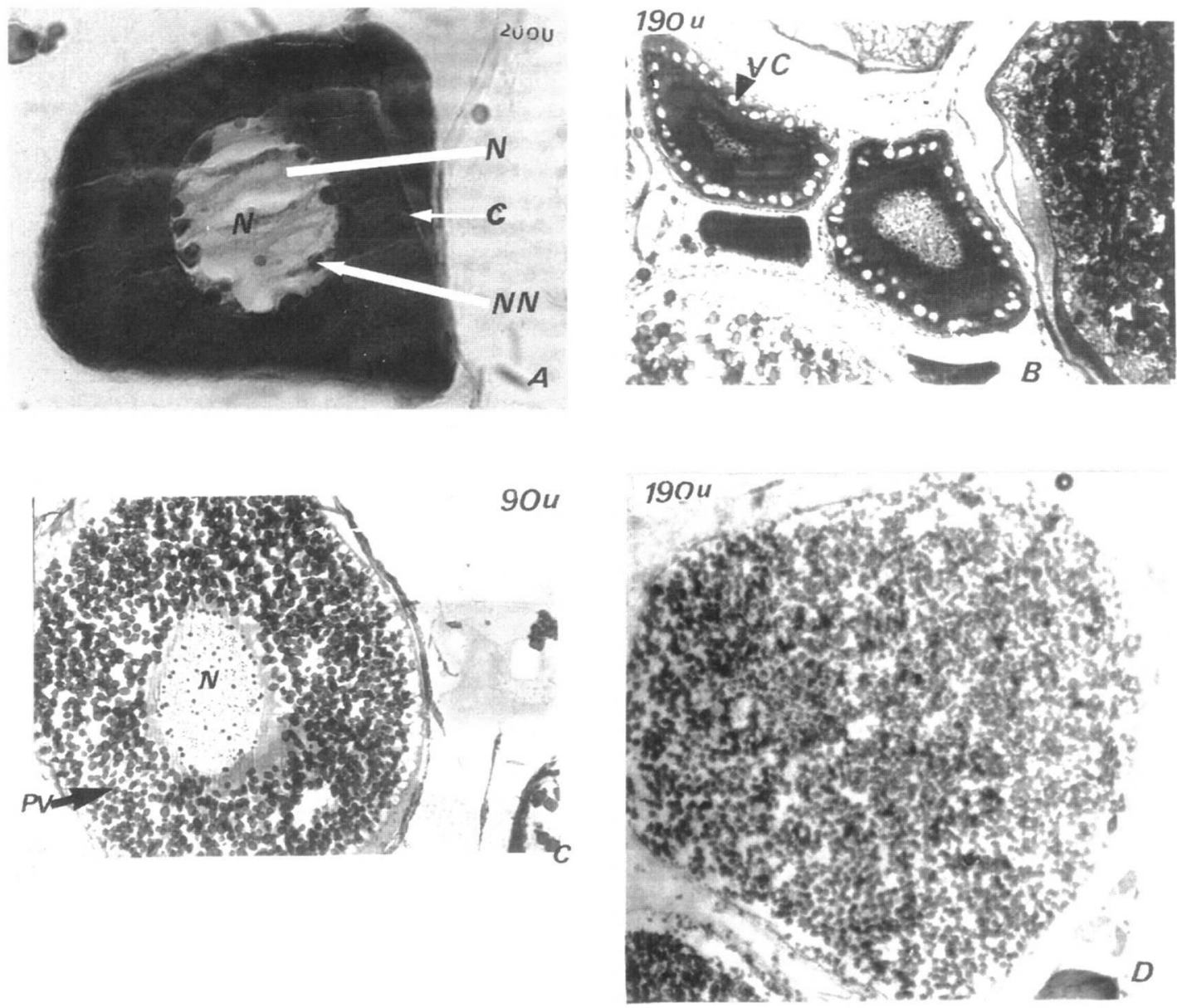

Figure 3 : Histologie de l'ovaire de Barbus holotaenia en ovogenèse. $A$ : follicule au stade II ; B : follicule au début du stade III ; C : follicule au stade IV ; D : ovocytes au stade $V$. ( $C$ : cytoplasme ; $N$ : noyau ; $N N$ : nucléole ; Vc : vacuole corticale (glycoprotéines); Pv : plaquette vitelline (lipoprotéines)).

Figure 3 : Histology of the ovary of B. holotaenia in ovogenesis. A : follicle in the stage II; $B$ : follicle in stage III ; $C$ : follicle in stage IV. $D$ : follicle in stage V. (N : nucleus ; NN : nucleolus ; $C$ : cytoplasm ; Vc : cortical vacuole ; PV : yolk plaquette). 


\section{DISCUSSION}

Les résultats obtenus permettent d'établir un schéma d'évolution globale des follicules et des ovocytes chez $B$. holotaenia conforme au modèle général décrit chez d'autres poissons téléostéens (LEGENDRE et JALABERT, 1988). Après maturation (du stade 0 au stade $\mathrm{V}$ ) : ovulation et ponte, la gonade passe au stade $\mathrm{VI}$ (atrésie) puis revient au stade 0 (repos) avant de recommencer un nouveau cycle sexuel. Ce phénomène se renouvelle durant toute la durée de vie de l'animal par divisions mitotiques successives.

La première phase de maturation (vitellogenèse endogène) consiste essentiellement en la mise en place de la machine métabolique indispensable à la croissance ultérieure de l'ovocyte. II s'agit notamment de la formation des vacuoles corticales au stade III et de celle des gouttelettes lipidiques et des plaquettes vitellines, sièges de synthèse d'acides aminés (NAGAHAMA, 1983) au stade IV. D'autres auteurs ont montré que l'apparition des vacuoles corticales se situe au stade II (BENGEN et al., 1991) et que les gouttelettes lipidiques se formaient après les vésicules vitellines (YAMAMOTO et al., 1965) et/ou après les vésicules et les plaquettes vitellines (YAMAMOTO, 1956).

La deuxième phase de maturation (vitellogenèse exogène) est observée vers la fin du stade III et se caractérise par la présence de nombreuses plaquettes vitellines orangiophiles. Ces plaquettes sont constituées en grande partie de vitellogénine, grosse molécule lipoprotéique synthétisée par le foie, circulant dans le sang et pénétrant dans l'ovocyte par micropinocytose (DROLLER et ROTH, 1966 ; ANDERSON, 1974 ; GUPTA et YAMAMOTO, 1972 ; WALLACE, 1978 ; LEGENDRE et JALABERT, 1988).

La présence chez $B$. holotaenia de polysaccharides acides dans certaines vacuoles corticales et de glycoprotéines dans d'autres permet d'établir une différence entre cette espèce et certaines espèces anadromes dont les ovocytes contiennent à la fois des polysaccharides acides et neutres (YAMAMOTO, 1956 ; BENGEN et al., 1991) ainsi que certains poissons marins dont les ovocytes ne contiennent que des polysaccharides neutres (YAMAMOTO, 1956).

\section{CONCLUSION}

Les résultats de l'examen microscopique des ovocytes ainsi que l'évolution de I'I.G.S. et l'observation d'alevins le long de différents cours d'eau permettent de penser que chez $B$. holotaenia, la vitellogenèse (endogène et exogène) et la ponte se déroulent durant la saison des pluies (Fig. 4). Ces observations sont en accord avec celles qui ont été faites par DE KIMPE (1964) dans le Luapula-Moero chez B. tropidolepis (BOULENGER, 1900) ; B. caudovittatus (BOULENGER, 1902) ; Raiamas moorei (BOULENGER, 1900) ; Acapoeta tanganicae (BOULENGER, 1910) et celles faites par MUTAMBUE et al. (1991) chez Opsaridium chrystyi (BOULENGER, 1920) dans le bassin de la Luki. Ce même type d'observations a été également fait en Côte d'lvoire par ALBARET (1982) chez Barbus sublineatus (DAGET, 1954) ; B. albabes (BLEEKER, 1863) ; et B. donaldsonsmithi (FLOWER, 1958) et dans d'autres pays d'Afrique Occidentale par PLANQUETTE et LEMASSON, (1975) chez Labeo parvus (BOULENGER, 1902) et chez L. senegalensis (VALENCIENNES, 1842) puis par NAWAR (1959), KRAMER (1973), PLANQUETTE et LEMASSON (1975) chez L. altivelis (PETERS, 1852).

Cette période d'activité sexuelle est concomitante avec la diminution de la température de l'eau (MUTAMBUE, 1984) et est conforme aux résultats des travaux expérimentaux de KIRSCHBAUM (1987) qui, à photopériode et température constantes, a induit la reproduction de plusieurs espèces de Mormyridae par l'action conjointe d'une imitation de la pluie, de l'élévation du niveau d'eau et de la baisse de la conductivité. Ces observations renforcent l'hypothèse selon laquelle la reproduction chez les poissons tropicaux est liée à la diminution de la température de l'eau (DAGET, 1952).

Les résultats de travaux réalisés en Afrique de l'Ouest indiquent que la durée et l'importance de crue ont une influence sur le nombre d'ovocytes émis et donc sur la fécondité globale du stock reproducteur (DANSOKO et al., 1976 ; ALBARET, 1982). La biologie de la reproduction de $B$. holotaenia étant jusqu'à présent inconnue, il semble 


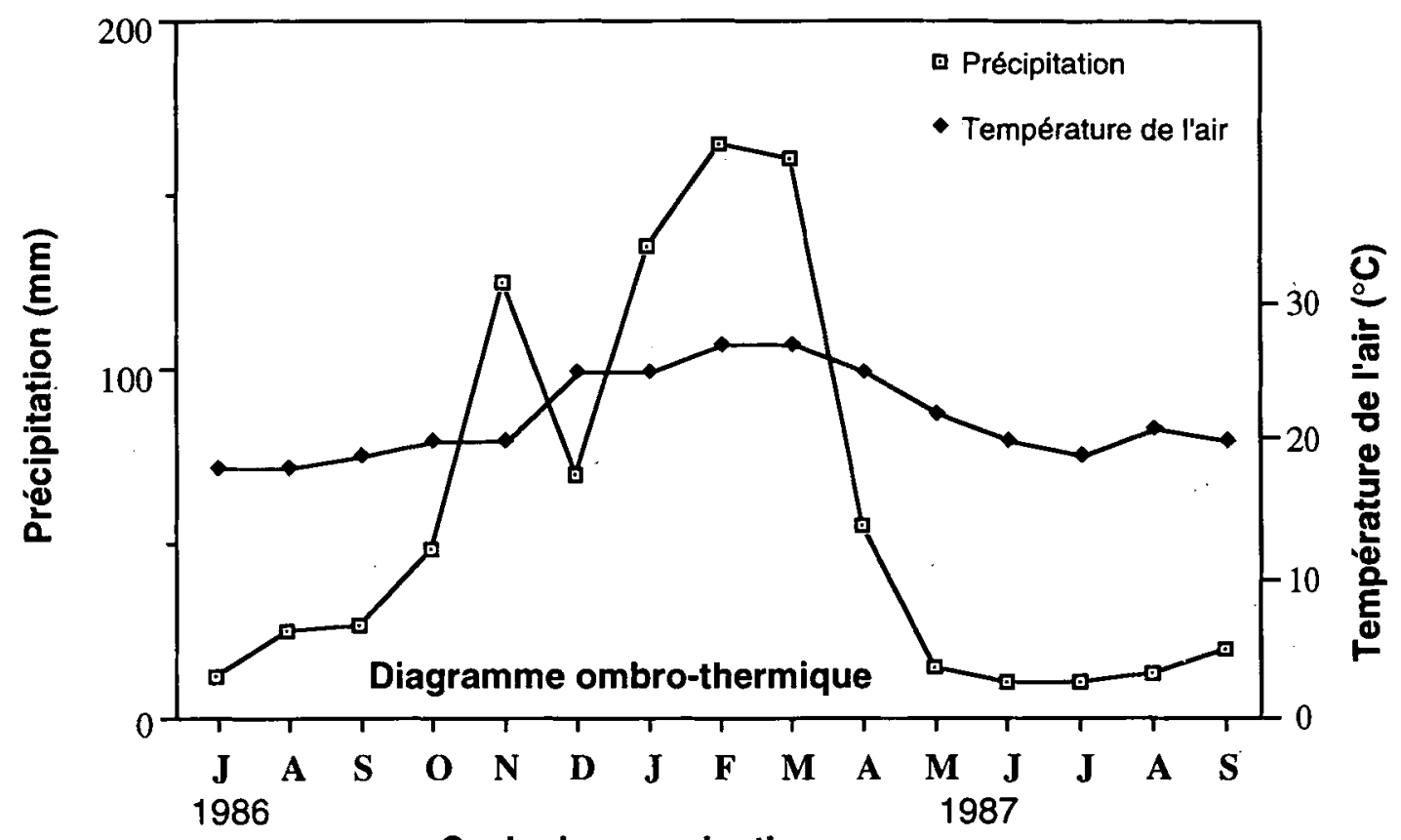

Cycle de reproduction

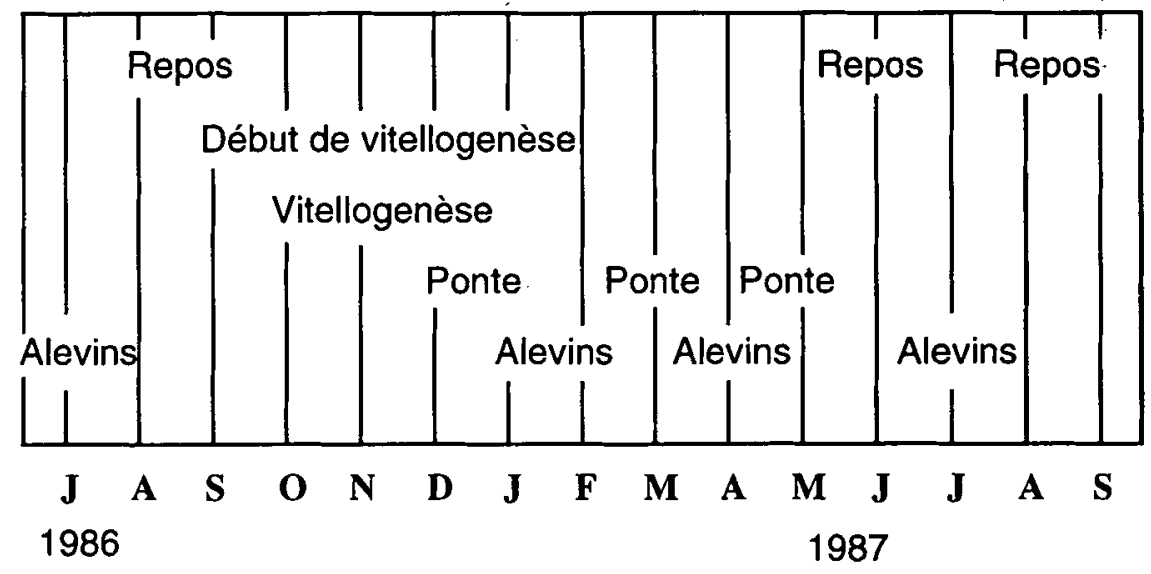

Figure 4 : Relation entre le cycle de reproduction de Barbus holotaenia femelle et les conditions climatiques du bassin de la Luki (données enregistrées à la station météorologique de I'INERA Luki).

Figure 4 : Relationship between the reproduction cycle of $B$. holotaenia female and the climatic conditions of the Luki river basin (data from the meteorological station of INERA Luki).

difficile d'évaluer l'impact et le rôle précis du facteur «hautes eaux» sur le cycle de reproduction de cette espèce. Mais on peut penser qu'il s'agit de l'adaptation du cycle de reproduction de Barbus holotaenia sur. le cycle général des eaux puisque c'est en cette saison que les disponibilités de nourriture pour l'élaboration des produits sexuels et pour l'alimentation de jeunes alevins sont maximales (BILLARD et BRETON, 1978 ; MOREAU et MOREAU, 1982 ; ROJAS-BELTRAN, 1984 ; MUTAMBUE et al., 1991). 


\section{BIBLIOGRAPHIE}

ABOUSSOUAN A., LAHAYE J., 1979. Les potentialités des populations ichtyologiques. Fécondité et ichtyoplancton. Cybium, 3 (6), 77-88.

ALBARET J.J., 1982. Reproduction et fécondité des poissons d'eau douce de Côted'Ivoire. Rev. Hydrobiol. Trop., 15 (4), 347-371.

ANDERSON E., 1974. Comparative aspects ultrastructure of female gamete. Int. Rev. Cytol., suppl. 4, 1-70.

BENECH V., QUENSIERE J., 1985. Stratégie de reproduction des poissons du Tchad en période de Tchad Normal (1966-1971). Rev. hydrobiol. trop., 18 (3), 227-244.

BENGEN G., KUGLER J., PEQUIGNOT J., 1991. Etudes des ovocytes d'Alosa alosa L. (Clupeidae) au cours de sa migration anadrome en Garonne. Cybium, 15 (3), 229-238.

BILLARD R., BRETON B., 1978. Rhythms of reproduction in teleost fish. In Thorpe J.E., Rhythmic activity in fishes, 31-53, Academic Press.

BOUGIS P., 1952. Rapport hépatosomatique et rapport gonadosomatique chez Mullus barbatus L. Bull. Soc. Zool. France, 74 (6), 326-330.

DAGET J., 1952. Mémoires sur la biologie des poissons de Niger. I. Biologie et croissance des espèces de genre Alestes. Bull. I.F.A.N., 14, 1, 191-225.

DAGET J., 1954. Les poissons du Niger Supérieur. Mém. I.F.A.N., 36, 391 p.

DANSOKO D., BREMAN H., DAGET J., 1976. Influence de la sécheresse sur les populations d'Hydrocinus dans le delta central du Niger. Cah. O.R.S.T.O.M., sér. Hydrobiol., vol. X, $\mathrm{n}^{\circ} 2,71-76$.

DE BONT A.F., 1948. Fish farming in the Belgian Congo. Nature London, 162, 998-999.

DE BONT A.F., 1950. Reproduction en étang du Tilapia melanopleura (Dum) et macrochir (Blgr). Compte rendu de la conférence piscicole anglo-belge, 1949 ; Communication $n^{\circ} 8,303-312$.

DE KIMPE P., 1964. Contribution à l'étude hydrobiologique du Luapula-Moero. Mus. Roy. Afr. Centr. Tervuren, Belgique. Annls. Sér. - in $8^{\circ}-$ Sc.- $n^{\circ} 128,238$ p.

DESTESCHE-GOMEZ F., RUWET J.C., 1967. Imprégnation et cohésion familiale chez Tilapia (Poissons Cichlidae). Annls. Soc. Roy. Zool. Belg., 3, 161-173.

DROLLER M.J., ROTH T.F., 1966. An electron microscope study of yolk formation during oogenesis in Lebistes reticulatus Guppyi. J. Cell. Biol., 28, 209-232.

DURAND J.R., LOUBENS G., 1970. Observation sur la sexualité et la reproduction des Alestes baremoze du bas Chari et du lac Tchad. Cah. O.R.S.T.O.M., Sér. Hydrobiol., 5 (2), 113-136.

FRANSSEN J., LAPERCHE J.P., 1980. Bioproductivité d'un étang à Sarotherodon niloticus et Tilapia tholoni dans la région de Kinshasa. Bull. Soc. Roy. Sc. Liège, 49 (11-12), 433-435.

GOSSE J.P., 1963. Le milieu aquatique et écologie des poissons dans la région de Yangambi. Annls. Mus. Roy. Afr. Centr. Sér. -in $8^{\circ}-$ Sc. Zool.- $n^{\circ} 116,115-247$.

GUPTA N.N., YAMAMOTO K., 1972. Electron microscope study on the fine structural changes in the oocytes of the goldfish, Carassius auratus, during yolk formation stage. Bull. Fac. Fish Hokkaido Univ., 22, 187-206.

KHALAF G., 1987. Le cycle sexuel de Capoeta damaxina (Cyprinidae) dans les cours d'eau libanais. Cybium, 11 (4), 395-402. 
KIRSCHBAUM F., 1984. Reproduction of weakly electric teleost : just another example of convergent development? Env. Biol. Fish., 10 (1-2), 3-14.

KIRSCHBAUM F., 1987. Reproduction and development of weakly electric fish, Pollimyrus isidori (Mormyridae, Teleostei) in captivity. Env. Biol. Fish., 20, 11-31.

KRAMER D. I., 1973. Preliminary investigations in the biology of Labeo in the Volta lake basin. Research Projet, Univ. of Ghana. Technical Report X, 38 p.

LEGENDRÉ M., JALABERT B., 1988. Physiologie de la reproduction. In LÉVÊQUE C., BRUTON M.N., SSENTONGO G.W., Biologie, Ecologie des poissons d'eau douce africains, 153-175, ORSTOM.

LOWE MC CONNELL R.H., 1964. The fish of the Rupununi savanna, district of British Guiana, South America. Part I. Ecology grouping of fish species and effects of seasonal cycle on the fish. J. Linn. Soc. (Zool.), 45, 103-144.

MATTHES H., 1964. Les poissons du lac Tumba et de la région d'lkela. Etude systématique et écologique. Annls. Mus. Roy. Afr. Centr. Tenvuren. Sér. in $8^{\circ}$. Vol. $126,203 \mathrm{p}$.

MOREAU J., MOREAU I., 1982. Etude du cycle annuel de la gamétogenèse chez Heterotis niloticus du lac Ivakona (zones des Pangalanes), Madagascar. Rev. Hydrobiol. trop., $15(3), 271-280$.

MUTAMBUE S., 1984. Contribution à l'étude de l'écologie de la rivière Luki (sous-affluent du fleuve Zaïre) : Bassin versant-Poissons. Thèse doct. $3^{\circ} \mathrm{Cycle}$, Univ. $P$. Sabatier/Toulouse, $213 p$.

MUTAMBUE S., 1992. Le bassin de la Luki et son aménagement. Systématique, biologie et écologie de sa faune piscicole. Thèse doct. Univ. P. Sabatier/Toulouse, $335 \mathrm{p}$.

MUTAMBUE S., EXBRAYAT J.M., GOUBIER J., 1991. Premières observations sur la biologie de la reproduction d'Opsaridium chrystyi (BOULENGER, 1920) (Cyprinidae) de la rivière Luki et de ses principaux affluents. Cybium, 15 (1), 23-33.

NAGAHAMA Y., 1983. The functional morphology of teleost gonads. In HOAR W.S., RENDALL D.J., DONALDSON E.M., Fish physiology, IX, 223-275, Acad. Press. $N-Y$, London.

NAWAR G., 1959. On the fecundity of Nile Catfish Synodontis schall (Bloch-Chneider, 1801). Sudan notes and records, 40, 139-141.

PLANQUETTE P., LEMASSON J., 1975. Le peuplement de poissons du Bandama blanc en pays Baoulé. Annls. Univ. Abidjan, sér. E. (Ecologie), 8 (1), 77-121.

ROJAS-BELTRAN R., 1984. Régimes alimentaires et croissance de quelques Siluriformes (Arius spp.) de Guyane. Résultats de la convention d'études portant sur les ressources halieutiques littorales de la Guyane. ISTPM Cayenne, 14-20.

RUWET J. C., 1962. La reproduction de Tilapia macrochir (Blg) et $T$. melanopleura (Dum) (Pisces Cichlidae) au lac de barrage de la Lufira (Katanga). Rev. Zool. et Bot. Africaines. LXVI, fasc. 3-4, 243-271.

RUWET J. C., 1963. Observation sur le comportement de Tilapia macrochir (Blg) (Pisces Cichlidae) au lac de retenue de la Lufira (Katanga). Behaviour, $X X, 3-4,242-250$.

RUWET J. C., 1968. Familial behaviour of Tilapia (Pisces Cichlidae) and its implications. Nature (London), 9, 997.

STAHL A., LERAY C., 1961. L'ovogenèse chez les poissons téléostéens. Arch. Anat. Microsc., 50 (2), 251-267.

WALLACE R.A., 1978. Oocyte growth in nonmammalian vertebrates. In the vertebrate ovary (R.E. Jones, ed.), Plenum New York, 469-502. 
Bull. Fr. Pêche Piscic. (1996) $340: 15-24 \quad-24-$

YAMAMOTO K., 1956. Studies on the formation of fish eggs. Ill. Localization of polysaccharides in oocytes of Liopsetta obscura. J. Fac. Sc. Hokkaido Univ. Ser., 4 (12), 391-399.

YAMAMOTO K., OOTA I., TAKANO K., ISHIKAWA T., 1965. Studies on the maturing process of the rainbow trout, Salmo gairdneri iridius. 1. Maturation of the ovary of a one-year-old fish. Bull. Jpn. Soc. Sc. Fish., 31, 123-132. 